\title{
Pivot Device
}

National Cancer Institute

\section{Source}

National Cancer Institute. Pivot Device. NCI Thesaurus. Code C50105.

A device designed to act as a center of rotation. 